\title{
Considerations regarding the micromagnetic resonance relaxometry technique for rapid label-free malaria diagnosis
}

\section{To the Editor:}

The imperfect diagnosis of malaria is a major impediment to the efforts to control and eliminate the disease ${ }^{1}$. The recent report by Peng et al. ${ }^{2}$ describing a novel, rapid and potentially low-cost micromagnetic resonance relaxometry technique for diagnosing malaria therefore addresses an important problem. Unfortunately, we believe that there is a major flaw in the authors' interpretation of the results of their measurements, which greatly impacts the estimates of the limits of detection of this technique.

Our most important concern relates to the interpretation of the relationship of the transverse relaxation rate $\left(R_{2}\right.$ index $)$ with the time after infection (shown in Fig. $4 \mathrm{~b}$ in the original paper ${ }^{2}$ ) and the parasitemia level (Figs. 2a and 4c). In these comparisons, the authors have used the interquartile range (IQR) of the measurements conducted using uninfected mice (far left scatter plot in Fig. 4b) as the 'baseline' level against which measurements of infected mice are compared. An inherent property of the IQR is that $25 \%$ of the data fall above the upper (75\%) level. Any diagnostic test evaluated against the $75 \%$ level would thus, by definition, result in $25 \%$ false-positive observations in uninfected individuals. We strongly believe that reliable detection of malaria infections with a new method should be assessed using a more stringent statistic, such as the $95 \%$ confidence level.

We have re-digitized the baseline (uninfected mice) data Peng et al. ${ }^{2}$ presented in Figure $4 \mathrm{~b}$ and have estimated the upper 95\% confidence level of the background $R_{2}$ index to be approximately $1.16 \mathrm{~s}^{-1}$. Assuming that reliable detection of infection is possible only if the mean signal is above this threshold, then the corresponding limit of detection in terms of 'parasitemia level' is approximately $1-2 \%$ (Fig. 4c), which is between 5,000- and 10,000-fold higher than the limit of detection claimed in the paper (10 parasites $/ \mu$ l of blood or $0.0002 \%$ parasitemia $)^{2}$.

Visual inspection of the data shown by Peng et al. ${ }^{2}$ in Figure $4 \mathrm{c}$ reveals that the $R_{2}$ signal does not change significantly below parasitemia levels of $1-2 \%$, strongly indicating that these levels are in fact indistinguishable from the background.

A similar concern pertains to the results presented by Peng et al. ${ }^{2}$ in
Figure 2a. Again, there is a large step between the background signal and the signal originating from the samples with the lowest parasite concentrations. If the background signal is indeed representative, then the measured signal will continuously approach the background signal at sufficiently low parasitemia levels. The observed discontinuity, rather than the expected continuous behavior, between uninfected samples and samples with extremely low parasitemia strongly indicates that an artifact (such as elevated methemoglobin levels) caused artificially high $R_{2}$ indices in the samples with undetectably low parasite numbers (such as those clearly shown in Fig. 4c for parasitemia levels below 1-2\%). Methemoglobin-which is formed, for example, over prolonged periods of blood sample storage or during malaria infection-contains paramagnetic high-spin $\mathrm{Fe}^{3+}$, leading to a higher $R_{2}$ (ref. 3).

In conclusion, our re-analyses of the data presented by Peng et al. ${ }^{2}$ indicate that, consistent with previous findings using conventional NMR techniques, the micro-NMR technique can only reliably determine parasitemia levels above $1-2 \%$ with $95 \%$ confidence 4 . It may therefore not represent a viable alternative to current tests.

\section{COMPETING FINANCIAL INTERESTS}

The authors declare no competing financial interests.

Stephan Karl ${ }^{1,2}$, Ivo Mueller ${ }^{1-3}$ \& Timothy G St Pierre ${ }^{4}$

${ }^{1}$ Population Health and Immunity Division, Walter and Eliza Hall Institute of Medical Research, Parkville, Victoria, Australia. ${ }^{2}$ Department of Medical Biology, Faculty of Medicine, Dentistry and Health Sciences, University of Melbourne, Parkville, Victoria, Australia. ${ }^{3}$ Center de Recerca en Salut Internacional de Barcelona (CRESIB), Barcelona, Spain. ${ }^{4}$ School of Physics, University of Western Australia, Perth, Western Australia, Australia.

e-mail:karl@wehi.edu.au.

1. MalERA Consultative Group on Diagnoses and Diagnostics. PLoS Med. 8, e1000396 (2011).

2. Peng, W.K. et al. Nat. Med. 20, 1069-1073 (2014).

3. Hänscheid, T. et al. Malar. J. 13, 285 (2014).

4. Karl, S., Gutierrez, L., House, M.J., Davis, T.M. \& St Pierre, T.G. Am. J. Trop. Med. Hyg. 85, 815-817 (2011).

\section{Han and Peng reply:}

Karl et al. ${ }^{1}$ question the validity of the analytical methodology used in our recent publication ${ }^{2}$ on the basis of the argument that, to achieve $95 \%$ confidence, the measured $R_{2}$ should be $\sim 2 \sigma$ away from the baseline (measurements from uninfected mice), where $\sigma$ is the s.d. of the baseline measurements. This argument would be correct if one were to make a single measurement from a mouse and use it for diagnostic determination, provided the underlying distribution follows a normal distribution ${ }^{3}$. However, as we stated in our paper $^{2}$, we do 4-8 samplings of blood from each mouse to obtain a distribution of readings for each individual mouse. Because our diagnostic categorization is based on a number of repeated measurements, the distribution of these values ('averaged $R_{2}$ ' values as shown in Fig. 1a) has smaller variation, allowing us to achieve statistical significance. These averaged $R_{2}$ values or 'true means' are significantly higher $(P<0.05)$ than the baseline (Fig. 1b; gray bar represents baseline uncertainty at the $95 \%$ confidence level), 